\title{
Filter ventilation and nicotine content of tobacco in cigarettes from Canada, the United Kingdom, and the United States
}

\author{
Lynn T Kozlowski, Nicholas Y Mehta, Christine T Sweeney, Stephen S Schwartz, \\ George P Vogler, Martin J Jarvis, Robert J West
}

\begin{abstract}
Objectives-The purpose was to determine filter ventilation and the nicotine content of tobacco and their contribution to machine-smoked yields of cigarettes from the United States, Canada, and the United Kingdom.

Methods-Ninety-two brands of cigarettes (32 American, 23 Canadian, and 37 British brands) were purchased at retail outlets in State College, Pennsylvania, United States, Toronto, Canada, and London, United Kingdom. A FIDUS FDT filter ventilation tester measured the percentage air-dilution from filter vents. High-pressure, liquid chromatography was used to measure the nicotine content of tobacco. Regression techniques were used to examine the contributions of tobacco nicotine content and filter ventilation to machine-smoked yields of tar, nicotine, and carbon monoxide (CO). Results-Ninety-four per cent of the American brands, 91\% of the Canadian brands, and $79 \%$ of British brands were ventilated. The total nicotine content of tobacco and percent nicotine (by weight of tobacco) averaged $10.2 \mathrm{mg}$ (standard error of the mean (SEM) 0.25 , range: 7.2 to 13.4 ) and $1.5 \%$ (SEM 0.03 , range 1.2 to 2 ) in the United States, $13.5 \mathrm{mg}$ (SEM 0.49, range: 8.0 to 18.3 ) and $1.8 \%$ (SEM 0.06, range: 1.0 to 2.4) in Canada, $12.5 \mathrm{mg}$ (SEM 0.33, range: 9 to 17.5 ) and $1.7 \%$ (SEM 0.04, range: 1.3 to 2.4 ) in the United Kingdom. Multiple regression analyses showed that ventilation was by far the largest factor influencing machine-smoked yields of tar, nicotine, and CO.

Conclusion-Filter ventilation appears to be the predominant method for reducing machine-smoked yields of tar, nicotine, and $\mathrm{CO}$ in three countries. However, some brands contain about twice as much nicotine (total content or percent nicotine) as do others, indicating that tobacco types or blends and tobacco casings can be used to manipulate nicotine content and nicotine delivery of cigarettes.

(Tobacco Control 1998;7:369-375)
\end{abstract}

Keywords: filter ventilation, nicotine content, cigarettes

Correspondence to:

Department of Biobehavioral

Health, 315 Health and

Human Development East,

Penn State University,

Penn State Univ
University Park,

University Park,

1tk1@psu.edu
Introduction

Outside of the cigarette industry, it is not widely known how much nicotine is in cigarette tobacco or how important filter ventilation is to the manipulation of the tar and nicotine yields of cigarettes. Standard machine tests for nicotine, tar, and carbon monoxide (CO) yields are very similar, though not identical, in the United States, Canada, and the United Kingdom. ${ }^{1}$ In each country $35 \mathrm{ml}, 2 \mathrm{~s}$ puffs are taken on cigarettes once a minute until similar butt lengths are reached. Tests of the nicotine content of tobacco have not been conducted or reported as part of the official tests of cigarettes. We know of only one peer-reviewed publication reporting the nicotine content of the tobacco from 15 popular cigarette brands, but this publication did not identify brand names. ${ }^{2}$ Filter vents can be made up of a region on the filter that has high porosity or discrete rings of holes around the circumference of the filter and starting at about $11-15 \mathrm{~mm}$ from the smoker end of the cigarette. Often these vents can only be detected if the filter covering is taken off the filter, held up to a bright light, and viewed through a magnifying glass. Percentage air dilution, or percentage filter ventilation, is defined as the percentage of a standard puff ( $2 \mathrm{~s}$ duration, $35 \mathrm{ml}$ ) that is air taken into the puff through filter vents. A cigarette with $0 \%$ filter ventilation produces a puff undiluted by filter vents. A cigarette with $80 \%$ filter ventilation produces a puff that is $80 \%$ air from vents and only $20 \%$ undiluted smoke.

Studies inside ${ }^{3}$ and outside ${ }^{4-8}$ the cigarette industry have found considerable evidence that (a) many smokers block filter vents with their fingers or lips and (b) thereby can increase their smoke exposure from these cigarettes. Smokers have been found to be generally unaware of the presence or function of vents, and such smokers are in need of a warning about filter vents. ${ }^{910}$ In smoking machine tests, Rickert and colleagues showed that vent-blocking on very low tar cigarettes was more important to increasing tar, nicotine, or CO yields than increasing puff volume or rate. ${ }^{11}$ When one is sucking mostly on air, more or bigger puffs do not get the smoker that much more smoke.

Here we report results of analyses for nicotine content of tobacco and filter ventilation performed on samples of cigarettes from three countries. This represents the first scientific publication of such data from an international sample. Such information may be valuable to a broad range of researchers and policy makers interested in the use and regula- 
Table 1 Standard tar, nicotine, and carbon monoxide yields ( $\mathrm{mg}$ ), total nicotine ( $m g$ ), percent nicotine (mg), distance of closest vents from smoker end of cigarette ( $\mathrm{mm}$ ), and filter ventilation levels (\%) for selected American cigarette brands

\begin{tabular}{|c|c|c|c|c|c|c|c|}
\hline Brand $^{\star}$ & $\operatorname{Tar}(m g) \dagger$ & Nicotine $(m g) t$ & $C O(m g) t$ & $\begin{array}{l}\text { Total nicotine } \\
(\mathrm{mg})\end{array}$ & $\begin{array}{l}\text { Percent nicotine } \\
\text { (mg) }\end{array}$ & $\begin{array}{l}\text { Closest vents } \\
(\mathrm{mm})\end{array}$ & $\begin{array}{l}\text { Percent ventilation } \\
\text { (SEM) }\end{array}$ \\
\hline Carlton SP & 1 & 0.1 & 2 & 8.7 & 1.7 & 15 & $77.6(0.32)$ \\
\hline Carlton $100 \mathrm{HP}$ & 1 & 0.1 & 1 & 10.4 & 1.6 & 14.5 & $82.5(0.29)$ \\
\hline Merit Ultima SP & 1 & 0.1 & 3 & 9.6 & 2.0 & 11.0 & $64.4(1.4)$ \\
\hline Carlton $100 \mathrm{SP}$ & 2 & 0.2 & 2 & 9.4 & 1.5 & 15.0 & $78.6(0.48)$ \\
\hline Now $100 \mathrm{SP}$ & 2 & 0.2 & 3 & 10.9 & 1.8 & 12.5 & $66.3(0.59)$ \\
\hline Merit UL SP & 4 & 0.4 & 5 & 9.8 & 1.6 & 11.5 & $49.0(0.54)$ \\
\hline Doral UL SP & 5 & 0.4 & 6 & 7.6 & 1.2 & 13.0 & $56.7(0.47)$ \\
\hline Benson \& Hedges Deluxe UL $100 \mathrm{HP}$ & 5 & 0.5 & 7 & 11.0 & 1.6 & 12.0 & $52.6(0.61)$ \\
\hline Virginia Slims UL $100 \mathrm{HP}$ & 5 & 0.5 & 6 & 10.4 & 1.6 & 12.0 & $55.6(0.72)$ \\
\hline Cambridge UL $100 \mathrm{SP}$ & 5 & 0.4 & 7 & 11.5 & 1.5 & 12.5 & $53.1(0.38)$ \\
\hline GPC UL SP & 5 & 0.4 & 7 & 8.0 & 1.3 & 15.0 & $47.9(0.67)$ \\
\hline Winston UL SP & 6 & 0.5 & 8 & 9.7 & 1.6 & 13.0 & $48.1(0.64)$ \\
\hline Merit HP & 7 & 0.6 & 9 & 9.4 & 1.5 & 11.0 & $34.1(0.71)$ \\
\hline Virginia Slim L $100 \mathrm{HP}$ & 8 & 0.7 & 9 & 9.4 & 1.4 & 12.0 & $39.7(0.46)$ \\
\hline Doral L SP & 8 & 0.6 & 10 & 8.3 & 1.4 & 12.5 & $18.9(0.59)$ \\
\hline Winston L SP & 9 & 0.7 & 11 & 10.3 & 1.5 & 12.0 & $24.8(0.56)$ \\
\hline GPC L SP & 9 & 0.6 & 11 & 7.2 & 1.2 & 15.0 & $23.7(0.34)$ \\
\hline Newport L SP & 9 & 0.7 & 11 & 11.4 & 1.7 & 14.0 & $21.8(0.62)$ \\
\hline Red Kamel L HPS & 10 & 0.8 & NA & 11.4 & 1.7 & 12.5 & $20.2(0.87)$ \\
\hline Marlboro L SP & 10 & 0.8 & 11 & 10.6 & 1.6 & 12.0 & $22.5(0.60)$ \\
\hline Basic L HP & 10 & 0.7 & 12 & 9.1 & 1.4 & 12.0 & $11.1(0.40)$ \\
\hline Camel L HP & 10 & 0.8 & 12 & 10.3 & 1.5 & 12.0 & $22.3(0.58)$ \\
\hline Kool Milds SP & 11 & 0.8 & 11 & 10.9 & 1.7 & 15.0 & $25.4(0.46)$ \\
\hline Marlboro Mediums $100 \mathrm{SP}$ & 12 & 0.9 & 13 & 11.2 & 1.5 & 12.5 & $19.1(0.31)$ \\
\hline Doral FF SP & 14 & 0.8 & 15 & 8.9 & 1.3 & 12.0 & $12.6(0.27)$ \\
\hline Winston FF SP & 14 & 1.0 & 14 & 10.5 & 1.5 & 15.0 & $11.7(0.87)$ \\
\hline Virginia Slims FF 100 SP & 15 & 1.1 & 13 & 11.7 & 1.6 & 12 & $19.9(0.87)$ \\
\hline Kool Filter HP & 16 & 1.1 & 14 & 13.0 & 1.9 & - & 0.0 \\
\hline Marlboro FF SP & 16 & 1.1 & 14 & 10.9 & 1.5 & 12.5 & $10.2(0.26)$ \\
\hline Newport FF HP & 17 & 1.3 & 19 & 13.4 & 1.9 & $\cdot$ & 0.0 \\
\hline Red Kamel FF HPS & 17 & 1.3 & NA & 11.6 & 1.6 & 15.0 & $21.8(0.99)$ \\
\hline Camel FF SP & 17 & 1.1 & 20 & 9.5 & 1.3 & 14.5 & $5.1(0.22)$ \\
\hline Mean (SE) & $8.8(0.90)$ & $0.67(0.06)$ & $9.6(0.87)$ & $10.2(0.25)$ & $1.5(0.03)$ & $12.9(0.25)$ & $34.3(4.2)$ \\
\hline
\end{tabular}

$\star \mathrm{UL}=$ Ultra light; $\mathrm{L}=\mathrm{Light} ; \mathrm{FF}=$ full flavour; $\mathrm{SP}=$ soft pack; HP = hard pack; unless designated 100, brand is king-size.

†Source: FTC yields from analyses on 1995 cigarettes in 1998; Federal Trade Commission. Tar, nicotine, and carbon monoxide of the smoke of 1206 varieties of domestic cigarettes, 1998.

$§$ Tar and nicotine yields were attained from advertisements. CO values not available.

$\mathrm{NA}=$ not available.

tion of cigarettes. (The filter ventilation results for the American cigarettes were previously published. ${ }^{12}$ ) American cigarettes are considered more likely to use "casings" (sauces and flavourings) than Canadian or British brands, so it was expected that the tobacco columns in American cigarettes would in general have lower nicotine contents. Our intention was to bring to light these elements of cigarette construction and to explore predictors of machine-smoked yields of tar, nicotine, and CO.

\section{Methods}

Ninety-two brands of cigarettes (32 American brands, 23 Canadian brands, and 37 British brands) were purchased at retail outlets in State College, Pennsylvania, United States, Toronto, Ontario, Canada, and London, England. British brands were selected to span the range of machine-smoked tar yields rather than be representative of sales.

VENTILATION TESTING

Each of the 92 cigarette packs was opened and immediately tested, unlit, for percent air dilution (filter ventilation), using an FDT ventilation tester (Fidus Instrument Corporation, Richmond, Virginia, USA). The ventilation testing device measures the amount of air passing through filter vent holes on an unlit cigarette during a $17.5 \mathrm{ml} / \mathrm{s}$ puff. The testing conditions maintained temperature at $23.9^{\circ} \mathrm{C}$ (standard error of the mean (SEM) 1) and $60 \%$ relative humidity (SEM 2). Twenty cigarettes were tested from each pack (mean (SEM) is reported). The results for any specific brand could be influenced by unique circumstances for the packs used. Manufacturing dates and temperature/humidity conditions of storage by retailers were unknown, and cigarette weights were not measured (as would likely be true for most cigarettes smoked by consumers). Cigarettes were all tested within one week of purchase. The brands were stored together in individual zip-lock bags until nicotine content testing, which was done within nine weeks of ventilation testing for the American brands, eight weeks of ventilation testing for the Canadian brands, and five weeks of ventilation testing for the British brands.

NICOTINE CONTENT MEASUREMENT

The nicotine assays were performed at the Behavioral Endocrinology Laboratory at Penn State University. The tobacco (literally, all the chopped brown material in the tobacco column) was first removed from five cigarettes for each brand and weighed and divided by five to give an estimate of the weight of a single cigarette. The tobacco was mixed thoroughly and the approximate equivalent of one cigarette's worth of tobacco was weighed out for nicotine analysis. Analytical procedures were closely modelled after those of Benowitz and colleagues. ${ }^{2}$ Nicotine was extracted by heating the tobacco in $20 \mathrm{ml}$ of $1 \mathrm{~N}$ hydrochloric acid for one hour in a hot water bath $\left(60-70^{\circ} \mathrm{C}\right)$. After the tubes stood overnight, they were mixed, and an aliquot $(1.5 \mathrm{ml})$ was 
Table 2 Standard tar, nicotine, and carbon monoxide yields ( $m g$ ), total nicotine ( $m g$ ), percent nicotine (mg), distance of closest vents from smoker end of cigarette $(\mathrm{mm})$, and filter ventilation levels (\%) for selected Canadian cigarette brands

\begin{tabular}{|c|c|c|c|c|c|c|c|}
\hline Brand $^{\star}$ & $\operatorname{Tar}(m g) t$ & $\begin{array}{l}\text { Nicotine } \\
(m g) t\end{array}$ & $C O(m g) t$ & $\begin{array}{l}\text { Total nicotine } \\
(\mathrm{mg})\end{array}$ & $\begin{array}{l}\text { Percent nicotine } \\
(m g)\end{array}$ & $\begin{array}{l}\text { Closest vents } \\
(\mathrm{mm})\end{array}$ & $\begin{array}{l}\text { Percent ventilation } \\
(S E M)\end{array}$ \\
\hline Medallion UM & 1 & 0.2 & 2 & 14.4 & 2.0 & 11.5 & $70.1(0.54)$ \\
\hline Craven UM & 1 & 0.1 & 1 & 11.2 & 1.7 & 12.5 & $79.0(0.47)$ \\
\hline Matinee EM & 4 & 0.4 & 5 & 13.7 & 1.9 & 12.5 & $60.6(0.88)$ \\
\hline Craven SM & 4 & 0.4 & 4 & 11.5 & 1.7 & 13.0 & $52.3(0.63)$ \\
\hline Craven UL & 6 & 0.6 & 6 & 12.4 & 1.7 & 13.0 & $50.6(0.67)$ \\
\hline du Maurier UL & 7 & 0.7 & 7 & 16.7 & 2.1 & 12.5 & $52.7(0.60)$ \\
\hline Export UL & 8 & 1.0 & 8 & 14.0 & 1.9 & 11.5 & $57.1(0.34)$ \\
\hline Craven EL & 8 & 0.9 & 8 & 11.9 & 1.7 & 13.0 & $37.5(0.56)$ \\
\hline du Maurier EL & 9 & 0.9 & 9 & 15.6 & 2.0 & 12.0 & $40.7(0.50)$ \\
\hline Craven L & 10 & 1.1 & 10 & 11.9 & 1.6 & 13.0 & $37.0(0.70)$ \\
\hline Export EL & 10 & 1.1 & 9 & 12.5 & 1.7 & 12.0 & $52.8(0.62)$ \\
\hline Export Mild & 11 & 1.1 & 11 & 12.3 & 1.6 & 12.0 & $52.4(0.76)$ \\
\hline du Maurier SM & 11 & 1.0 & 12 & 13.5 & 1.7 & 13.0 & $31.2(0.36)$ \\
\hline Players EL & 11 & 1.2 & 11 & 18.3 & 2.3 & 12.0 & $42.2(0.37)$ \\
\hline du Maurier L & 12 & 1.2 & 12 & 17.3 & 2.2 & 12.5 & $30.2(0.29)$ \\
\hline Export L & 12 & 1.1 & 12 & 13.6 & 1.8 & 12.0 & $40.0(0.95)$ \\
\hline Players L King & 13 & 1.3 & 14 & 14.5 & 1.9 & 13.0 & $16.4(0.21)$ \\
\hline Craven FF & 13 & 1.2 & 13 & 11.6 & 1.7 & 13.5 & $14.6(0.28)$ \\
\hline Players L Smooth & 13 & 1.3 & 14 & 16.3 & 2.0 & 13.0 & $14.8(0.21)$ \\
\hline Export Med & 14 & 1.2 & 14 & 12.1 & 1.6 & 12.0 & $21.2(0.60)$ \\
\hline du Maurier & 15 & 1.3 & 16 & 15.4 & 1.9 & $\cdot$ & 0.0 \\
\hline Players FF & 16 & 1.4 & 17 & 8.0 & 1.0 & - & 0.0 \\
\hline Export FF & 16 & 1.4 & 17 & 12.6 & 1.4 & 12.5 & $15.7(0.36)$ \\
\hline Mean (SE) & $9.8(0.91)$ & $0.96(0.08)$ & $10.1(0.94)$ & $13.5(0.49)$ & $1.8(0.06)$ & $12.5(0.12)$ & $37.8(4.4)$ \\
\hline
\end{tabular}

${ }^{\star} \mathrm{UM}=$ Ultra mild, $\mathrm{EM}=$ Extra mild, $\mathrm{SM}=$ Special mild, $\mathrm{UL}=$ Ultra light, $\mathrm{EL}=$ Extra light, L = Light, FF = Full Flavour. † Source: the cigarette packages.

removed and transferred to a microcentrifuge tube and spun at $14000 \mathrm{rpm}$ for 20 minutes; $100 \mu \mathrm{l}$ of the top layer (supernatant) was transferred to a high-performance liquid chromatography (HPLC) vial. $900 \mu \mathrm{l} \quad 0.15 \mathrm{~N}$ ammonium hydroxide was added to the supernatant and vortexed for $5 \mathrm{~s}$. Nicotine concentrations were measured by HPLC (Waters 600E pump with a Waters Lambda-Max Model UV/Vis detector set at $254 \mathrm{~nm}$ ). A Zorbax chromatography column was used, with a mobile phase of $70 \%$ methanol and $30 \%$ aqueous solution containing $1.0 \%$ triethylamine adjusted to a

Table 3 Standard tar, nicotine, and carbon monoxide yields ( $\mathrm{mg}$ ), total nicotine ( $\mathrm{mg}$ ), percent nicotine (mg), distance of closest vents from smoker end of cigarette $(\mathrm{mm})$, and filter ventilation levels (\%) for selected United Kingdom cigarette brands

\begin{tabular}{|c|c|c|c|c|c|c|c|}
\hline Brand $^{\star}$ & $\operatorname{Tar}(m g) t$ & Nicotine $(m g) t$ & $C O(m g) t$ & $\begin{array}{l}\text { Total nicotine } \\
(\mathrm{mg})\end{array}$ & $\begin{array}{l}\text { Percent nicotine } \\
\text { (mg) }\end{array}$ & $\begin{array}{l}\text { Closest vents } \\
(\mathrm{mm})\end{array}$ & $\begin{array}{l}\text { Percent ventilation } \\
\text { (SEM) }\end{array}$ \\
\hline Silk Cut Ultra & 1 & 0.2 & 1 & 15.7 & 2.4 & 12.0 & $84.2(0.25)$ \\
\hline Benson \& Hedges UL & 1 & 0.2 & 1 & 14.6 & 2.2 & 13.0 & $82.2(0.22)$ \\
\hline Silk Cut Extra Mild & 3 & 0.3 & 3 & 10.8 & 1.7 & 12.5 & $69.5(0.29)$ \\
\hline John Player SK UL & 3 & 0.3 & 3 & 10.7 & 1.7 & 15.0 & $62.5(0.71)$ \\
\hline Lambert \& Butler L & 5 & 0.5 & 7 & 11.0 & 1.7 & 16.0 & $31.4(0.41)$ \\
\hline Silk Cut & 5 & 0.5 & 5 & 13.8 & 1.9 & 13.0 & $51.2(0.57)$ \\
\hline John Player Special L & 6 & 0.5 & 9 & 11.1 & 1.8 & 16.5 & $15.5(0.54)$ \\
\hline Rothmans L & 6 & 0.6 & 7 & 11.3 & 1.7 & 10.5 & $56.3(0.51)$ \\
\hline Benson \& Hedges L & 6 & 0.6 & 6 & 13.3 & 1.8 & 11.0 & $52.6(1.17)$ \\
\hline Marlboro L & 6 & 0.6 & 7 & 10.2 & 1.7 & 10.5 & $44.9(0.47)$ \\
\hline Dunhill L & 7 & 0.7 & 8 & 12.4 & 1.7 & 14.0 & $36.4(1.09)$ \\
\hline Rothman Royal L & 7 & 0.7 & 7 & 14.3 & 1.9 & 11.5 & $38.4(0.65)$ \\
\hline Dorchester Extra Mild & 8 & 0.7 & 8 & 12.2 & 1.6 & 11.0 & $40.1(0.54)$ \\
\hline Embassy Mild & 8 & 0.8 & 9 & 11.9 & 1.6 & 13.5 & $23.0(0.81)$ \\
\hline Craven Special Mild & 8 & 0.7 & 9 & 9.9 & 1.4 & 13.0 & $34.8(0.78)$ \\
\hline Knights Mild & 8 & 0.7 & 10 & 10.2 & 1.5 & 12.0 & $29.5(0.54)$ \\
\hline Consulate Menthol & 9 & 0.7 & 8 & 12.1 & 1.5 & 15.0 & $36.3(0.65)$ \\
\hline Berkeley SK L & 9 & 0.8 & 9 & 13.5 & 1.6 & 12.0 & $40.6(0.66)$ \\
\hline Berkeley SK Menthol & 9 & 0.7 & 9 & 13.9 & 1.7 & 12.0 & $47.5(0.45)$ \\
\hline Benson \& Hedges SK L & 9 & 0.8 & 10 & 13.9 & 1.7 & 12.5 & $38.4(0.65)$ \\
\hline Embassy & 11 & 1.0 & 12 & 10.1 & 1.6 & 11.5 & $8.0(0.33)$ \\
\hline John Player Special & 11 & 1.1 & 13 & 11.9 & 1.7 & NA & 0.0 \\
\hline John Player SK & 12 & 1.1 & 14 & 14.4 & 1.7 & NA & 0.0 \\
\hline Lambert \& Butler & 12 & 1.1 & 13 & 13.0 & 1.7 & NA & 0.0 \\
\hline Dorchester & 12 & 0.9 & 15 & 9.0 & 1.3 & 10.5 & $17.1(0.24)$ \\
\hline Berkeley SK & 12 & 1.0 & 16 & 13.3 & 1.6 & NA & 0.0 \\
\hline Benson \& Hedges Special Filter & 12 & 1.0 & 14 & 12.2 & 1.6 & 11.0 & $10.3(0.28)$ \\
\hline Benson \& Hedges SK & 12 & 1.0 & 15 & 15.2 & 1.9 & NA & 0.0 \\
\hline Regal & 12 & 1.2 & 15 & 10.6 & 1.5 & NA & 0.0 \\
\hline Embassy No 1 & 12 & 1.0 & 15 & 12.5 & 1.7 & NA & 0.0 \\
\hline Raffles 100 & 13 & 1.1 & 15 & 17.5 & 2.0 & 11.0 & $5.2(0.09)$ \\
\hline Benington SK & 13 & 0.8 & 17 & 12.2 & 1.4 & NA & 0.0 \\
\hline Knightsbridge SK & 13 & 1.1 & 14 & 15.9 & 1.7 & 12.5 & $22.9(0.56)$ \\
\hline Rothman Royals & 13 & 1.1 & 12 & 13.5 & 1.8 & 11.5 & $14.6(0.20)$ \\
\hline Benington & 13 & 1.1 & 14 & 10.0 & 1.4 & 13.0 & $30.2(0.54)$ \\
\hline Marlboro & 13 & 0.9 & 13 & 9.6 & 1.3 & 11.0 & $19.5(0.26)$ \\
\hline Rothmans & 13 & 1.2 & 13 & 14.3 & 1.8 & 12.5 & $26.6(0.41)$ \\
\hline Mean (SE) & $9.1(0.59)$ & $0.78(0.05)$ & $10.3(0.70)$ & $12.5(0.33)$ & $1.7(0.22)$ & & $28.9(3.9)$ \\
\hline
\end{tabular}

$\star \mathrm{UL}=$ Ultra light; $\mathrm{L}=$ Light; SK = Super King.

$\mathrm{NA}=$ not applicable.

†Source: Laboratory of the Government Chemist, Jan-Dec 1996. (Cigarette packages). 
Table 4 Correlation coefficients for key variables (95\% confidence intervals) (upper diagonal $=$ United States; lower diagonal = Canada)

\begin{tabular}{|c|c|c|c|c|c|c|c|}
\hline & Standard tar & Standard nicotine & Standard $C O$ & Percent ventilation & Total nicotine & Percent nicotine & Filter weight \\
\hline Standard tar & 1.00 & $\begin{array}{l}0.98 \\
(0.96 \text { to } 0.99)\end{array}$ & $\begin{array}{l}0.97 \\
(0.93 \text { to } 0.99)\end{array}$ & $\begin{array}{l}-0.92 \\
(-0.96 \text { to }-0.84)\end{array}$ & $\begin{array}{l}0.44 \\
(0.10 \text { to } 0.68)\end{array}$ & $\begin{array}{l}-0.07 \\
(-0.41 \text { to } 0.29)\end{array}$ & $\begin{array}{l}-0.79 \\
(-0.89 \text { to }-0.61)\end{array}$ \\
\hline Standard nicotine & $\begin{array}{l}0.97 \\
(0.93 \text { to } 0.99)\end{array}$ & 1.00 & $\begin{array}{l}0.95 \\
(0.90 \text { to } 0.98)\end{array}$ & $\begin{array}{l}-0.90 \\
(-0.95 \text { to }-0.81)\end{array}$ & $\begin{array}{l}0.51 \\
(0.20 \text { to } 0.73)\end{array}$ & $\begin{array}{l}-0.01 \\
(-0.35 \text { to } 0.34)\end{array}$ & $\begin{array}{l}-0.77 \\
(-0.88 \text { to }-0.58)\end{array}$ \\
\hline Standard CO & $\begin{array}{l}0.99 \\
(0.98 \text { to } 0.99)\end{array}$ & $\begin{array}{l}0.95 \\
(0.89 \text { to } 0.98)\end{array}$ & 1.00 & $\begin{array}{l}-0.95 \\
(-0.98 \text { to }-0.90)\end{array}$ & $\begin{array}{l}0.32 \\
(-0.05 \text { to } 0.61)\end{array}$ & $\begin{array}{l}-0.16 \\
(-0.49 \text { to } 0.21)\end{array}$ & $\begin{array}{l}-0.73 \\
(-0.87 \text { to }-0.51)\end{array}$ \\
\hline Percent ventilation & $\begin{array}{l}-0.90 \\
(-0.96 \text { to }-0.78)\end{array}$ & $\begin{array}{l}-0.84 \\
(-0.93 \text { to }-0.65)\end{array}$ & $\begin{array}{l}-0.92 \\
(-0.97 \text { to } 0.83)\end{array}$ & 1.00 & $\begin{array}{l}-0.33 \\
(-0.61 \text { to } 0.03)\end{array}$ & $\begin{array}{l}0.11 \\
(-0.25 \text { to } 0.44)\end{array}$ & $\begin{array}{l}0.66 \\
(0.40 \text { to } 0.82)\end{array}$ \\
\hline Total nicotine & $\begin{array}{l}0.00 \\
(-0.41 \text { to } 0.41)\end{array}$ & $\begin{array}{l}0.08 \\
(-0.34 \text { to } 0.48)\end{array}$ & $\begin{array}{l}0.01 \\
(-0.41 \text { to } 0.42)\end{array}$ & $\begin{array}{l}0.06 \\
(-0.36 \text { to } 0.46)\end{array}$ & 1.00 & $\begin{array}{l}0.70 \\
(0.47 \text { to } 0.84)\end{array}$ & $\begin{array}{l}-0.23 \\
(-0.53 \text { to } 0.13)\end{array}$ \\
\hline Percent nicotine & $\begin{array}{l}-0.27 \\
(-0.61 \text { to } 0.16)\end{array}$ & $\begin{array}{l}-0.17 \\
(-0.55 \text { to } 0.26)\end{array}$ & $\begin{array}{l}-0.27 \\
(-0.61 \text { to } 0.16)\end{array}$ & $\begin{array}{l}0.28 \\
(-0.15 \text { to } 0.62)\end{array}$ & $\begin{array}{l}0.94 \\
(0.85 \text { to } 0.97)\end{array}$ & 1.0 & $\begin{array}{l}0.26 \\
(-0.10 \text { to } 0.56)\end{array}$ \\
\hline Filter weight & $\begin{array}{l}-0.46 \\
(-0.74 \text { to }-0.06)\end{array}$ & $\begin{array}{l}-0.45 \\
(-0.73 \text { to } 0.05)\end{array}$ & $\begin{array}{l}-0.43 \\
(-0.72 \text { to } 0.02)\end{array}$ & $\begin{array}{l}0.30 \\
(-0.13 \text { to } 0.63)\end{array}$ & $\begin{array}{l}0.46 \\
(0.05 \text { to } 0.73)\end{array}$ & $\begin{array}{l}0.52 \\
(0.14 \text { to } 0.77)\end{array}$ & 1.0 \\
\hline
\end{tabular}

Table 5 Correlation coefficients for key variables (95\% confidence intervals) (upper diagonal $=$ United Kingdom; lower diagonal = all combined)

\begin{tabular}{|c|c|c|c|c|c|c|c|}
\hline & Standard tar $(m g)$ & $\begin{array}{l}\text { Standard nicotine } \\
(m g)\end{array}$ & Standard CO (mg) & Percent ventilation & Total nicotine & Percent nicotine & Filter weight \\
\hline Standard tar & 1.00 & $\begin{array}{l}0.96 \\
(0.92 \text { to } 0.98)\end{array}$ & $\begin{array}{l}0.97 \\
(0.93 \text { to } 0.98)\end{array}$ & $\begin{array}{l}-0.86 \\
(-0.93 \text { to }-0.74)\end{array}$ & $\begin{array}{l}0.03 \\
(-0.29 \text { to } 0.35)\end{array}$ & $\begin{array}{l}-0.49 \\
(-0.70 \text { to }-0.20)\end{array}$ & $\begin{array}{l}-0.45 \\
(-0.68 \text { to }-0.15)\end{array}$ \\
\hline Standard nicotine & $\begin{array}{l}0.94 \\
(0.91 \text { to } 0.96)\end{array}$ & 1.00 & $\begin{array}{l}0.91 \\
(0.84 \text { to } 0.96)\end{array}$ & $\begin{array}{l}-0.85 \\
(-0.92 \text { to }-0.73)\end{array}$ & $\begin{array}{l}0.09 \\
(-0.24 \text { to } 0.40)\end{array}$ & $\begin{array}{l}-0.40 \\
(-0.64 \text { to }-0.08)\end{array}$ & $\begin{array}{l}-0.51 \\
(-0.71 \text { to }-0.22)\end{array}$ \\
\hline Standard CO & $\begin{array}{l}0.97 \\
(0.95 \text { to } 0.98)\end{array}$ & $\begin{array}{l}0.89 \\
(0.83 \text { to } 0.93)\end{array}$ & 1.00 & $\begin{array}{l}-0.92 \\
(-0.96 \text { to }-0.84)\end{array}$ & $\begin{array}{l}-0.00 \\
(-0.33 \text { to } 0.32)\end{array}$ & $\begin{array}{l}-0.50 \\
(-0.71 \text { to }-0.20)\end{array}$ & $\begin{array}{l}-0.35 \\
(-0.61 \text { to }-0.03)\end{array}$ \\
\hline Percent ventilation & $\begin{array}{l}-0.86 \\
(-0.91 \text { to }-0.80)\end{array}$ & $\begin{array}{l}-0.78 \\
(-0.85 \text { to }-0.68)\end{array}$ & $\begin{array}{l}-0.92 \\
(-0.95 \text { to }-0.88)\end{array}$ & 1.00 & $\begin{array}{l}0.05 \\
(-0.28 \text { to } 0.36)\end{array}$ & $\begin{array}{l}0.42 \\
(0.11 \text { to } 0.65)\end{array}$ & $\begin{array}{l}0.37 \\
(0.05 \text { to } 0.62)\end{array}$ \\
\hline Total nicotine & $\begin{array}{l}0.16 \\
(-0.04 \text { to } 0.36)\end{array}$ & $\begin{array}{l}0.33 \\
(0.13 \text { to } 0.50)\end{array}$ & $\begin{array}{l}0.10 \\
(-0.11 \text { to } 0.30)\end{array}$ & $\begin{array}{l}-0.04 \\
(-0.24 \text { to } 0.17)\end{array}$ & 1.00 & $\begin{array}{l}0.73 \\
(0.53 \text { to } 0.85)\end{array}$ & $\begin{array}{l}0.25 \\
(-0.08 \text { to } 0.53)\end{array}$ \\
\hline Percent nicotine & $\begin{array}{l}-0.20 \\
(-0.39 \text { to } 0.001)\end{array}$ & $\begin{array}{l}-0.04 \\
(-0.24 \text { to } 0.17)\end{array}$ & $\begin{array}{l}-0.27 \\
(-0.45 \text { to }-0.06)\end{array}$ & $\begin{array}{l}0.26 \\
(0.06 \text { to } 0.44)\end{array}$ & $\begin{array}{l}0.82 \\
(0.75 \text { to } 0.88)\end{array}$ & 1.0 & $\begin{array}{l}0.37 \\
(0.05 \text { to } 0.62)\end{array}$ \\
\hline Filter weight & $\begin{array}{l}-0.54 \\
(-0.67 \text { to }-0.37)\end{array}$ & $\begin{array}{l}-0.63 \\
(-0.74 \text { to }-0.49)\end{array}$ & $\begin{array}{l}-0.43 \\
(-0.59 \text { to }-0.25)\end{array}$ & $\begin{array}{l}0.35 \\
(0.15 \text { to } 0.51)\end{array}$ & $\begin{array}{l}-0.32 \\
(-0.49 \text { to }-0.12)\end{array}$ & $\begin{array}{l}-0.05 \\
(-0.25 \text { to } 0.16)\end{array}$ & 1.0 \\
\hline
\end{tabular}

$\mathrm{pH}$ of 8 with acetic acid at a flow rate of $0.7 \mathrm{ml}$ per minute. The retention time for nicotine was about 9.5 minutes. An external standard of nicotine was used, which gave a linear standard curve over the entire range $(0-150 \mu \mathrm{g} / \mathrm{ml})$ of observed concentrations. The nicotine standard was (S)-(-)-nicotine (98\%) (Aldrich Chemical Company, Milwaukee, Wisconsin, USA) in a reagent of one part $1 \mathrm{~N} \mathrm{HCl}$ to nine parts $0.15 \mathrm{~N}$ ammonium hydroxide.

STATISTICAL ANALYSES

Values for individual brands are reported with descriptive statistics for each country. Pearson correlations and multiple regression analyses were performed. Because of high intercorrelations among the key variables, several multiple regression models are reported, to explore the contributions of predictor variables. Standardised regression coefficients are not good indicators of the importance of independent variables when there are high intercorrelations among the independent variables. ${ }^{13}$

\section{Results}

See tables 1, 2, and 3 for data for each brand.

FILTER VENTILATION

A total of $94 \%$ of the American brands, $91 \%$ of the Canadian brands, and $79 \%$ of the British brands were ventilated. Mean percentage ventilation for the ventilated brands ranged from $5.1 \%$ to $84.2 \%$ across the three countries. Brands with machine-smoked yields of $1 \mathrm{mg}$ tar averaged $75 \%$ air diluted by filter vents in the United States and Canada, and 83\% diluted in the United Kingdom. All American and Canadian brands with machine-smoked yields of $14 \mathrm{mg}$ or less of tar were ventilated; all British brands with machine-smoked yields of $9 \mathrm{mg}$ or less of tar were ventilated.

\section{NICOTINE CONTENT}

Analysis of variance with Bonferroni comparisons showed that the American cigarettes averaged less nicotine (total or percent) than either British or Canadian cigarettes $(\mathrm{p}<0.008$ in all cases).

Table 6 Beta weights (95\% confidence intervals) for multiple regression equations predicting standard tar and nicotine yields of American cigarettes from percent ventilation, percent nicotine in tobacco, nicotine content per cigarette, and filter weight. Each column describes a different regression equation.

\begin{tabular}{|c|c|c|c|c|c|c|c|c|}
\hline \multirow[b]{2}{*}{ Percent ventilation } & \multicolumn{4}{|c|}{ Predicting tar yields } & \multicolumn{4}{|c|}{ Predicting nicotine yields } \\
\hline & $\begin{array}{l}-0.87 \\
(0.73 \text { to } 1.01)\end{array}$ & $\begin{array}{l}-0.92 \\
(-1.06 \text { to }-0.78)\end{array}$ & $\begin{array}{l}-0.79 \\
(-0.95 \text { to }-0.63)\end{array}$ & $\begin{array}{l}-0.65 \\
(-0.79 \text { to }-0.51)\end{array}$ & $\begin{array}{l}-0.82 \\
(-0.96 \text { to }-0.68)\end{array}$ & $\begin{array}{l}-0.91 \\
(-1.07 \text { to }-0.75)\end{array}$ & $\begin{array}{l}-0.74 \\
(-0.89 \text { to }-0.59)\end{array}$ & $\begin{array}{l}-0.61 \\
(-0.75 \text { to }-0.47)\end{array}$ \\
\hline $\begin{array}{l}\text { Percent nicotine } \\
\text { (mg nicotine/mg } \\
\text { tobacco) }\end{array}$ & NA & $\begin{array}{l}0.03 \\
(-0.11 \text { to } 0.17)\end{array}$ & $\begin{array}{l}-0.22 \\
(-0.42 \text { to }-0.02)\end{array}$ & $\begin{array}{l}-0.06 \\
(-0.24 \text { to } 0.12)\end{array}$ & NA & $\begin{array}{l}0.09 \\
(-0.07 \text { to } 0.25)\end{array}$ & $\begin{array}{l}-0.23 \\
(-0.43 \text { to }-0.03)\end{array}$ & $\begin{array}{l}-0.09 \\
(-0.29 \text { to } 0.11)\end{array}$ \\
\hline $\begin{array}{l}\text { Total nicotine } \\
\text { (nicotine// } \\
\text { cigarette }(\mathrm{mg}))\end{array}$ & $\begin{array}{l}0.15 \\
(0.01 \text { to } 0.29)\end{array}$ & NA & $\begin{array}{l}0.33 \\
(0.11 \text { to } 0.55)\end{array}$ & $\begin{array}{l}0.20 \\
(0.02 \text { to } 0.38)\end{array}$ & $\begin{array}{l}0.24 \\
(0.10 \text { to } 0.38)\end{array}$ & NA & $\begin{array}{l}0.43 \\
(0.21 \text { to } 0.65)\end{array}$ & $\begin{array}{l}0.31 \\
(0.11 \text { to } 0.51)\end{array}$ \\
\hline Filter weight (mg) & NA & NA & NA & $\begin{array}{l}-0.31 \\
(-0.45 \text { to } 0.17)\end{array}$ & NA & NA & NA & $\begin{array}{l}-0.28 \\
(-0.44 \text { to }-0.12)\end{array}$ \\
\hline Intercept & 9.64 & 14.39 & 11.4 & 17.71 & 0.48 & 0.87 & 0.61 & 0.99 \\
\hline RMS error & 1.91 & 2.05 & 1.81 & 1.44 & 0.13 & 0.15 & 0.12 & 0.10 \\
\hline$R^{2}($ all $\mathrm{p}<0.0001)$ & 0.87 & 0.85 & 0.89 & 0.93 & 0.87 & 0.82 & 0.89 & 0.92 \\
\hline
\end{tabular}

$\mathrm{NA}=$ not applicable. 
Table 7 Beta weights (95\% confidence intervals) for multiple regression equations predicting standard tar and nicotine yields of British cigarettes from percent ventilation, percent nicotine in tobacco, nicotine content per cigarette, and filter weight. Each column describes a different regression equation.

\begin{tabular}{|c|c|c|c|c|c|c|c|c|}
\hline \multirow[b]{2}{*}{ Percent ventilation } & \multicolumn{4}{|l|}{ Predicting tar yields } & \multicolumn{4}{|c|}{ Predicting nicotine yields } \\
\hline & $\begin{array}{l}-0.79 \\
(-0.97 \text { to }-0.61)\end{array}$ & $\begin{array}{l}-0.86 \\
(-1.04 \text { to }-0.68)\end{array}$ & $\begin{array}{l}-0.65 \\
(-0.81 \text { to }-0.49)\end{array}$ & $\begin{array}{l}-0.60 \\
(-0.76 \text { to }-0.44)\end{array}$ & $\begin{array}{l}-0.83 \\
(-1.03 \text { to }-0.63)\end{array}$ & $\begin{array}{l}-0.86 \\
(-1.04 \text { to }-0.68)\end{array}$ & $\begin{array}{l}-0.70 \\
(-0.88 \text { to }-0.52)\end{array}$ & $\begin{array}{l}-0.63 \\
(-0.81 \text { to }-0.45)\end{array}$ \\
\hline $\begin{array}{l}\text { Percent nicotine } \\
\text { (mg nicotine/mg } \\
\text { tobacco) }\end{array}$ & $\begin{array}{l}-0.16 \\
(-0.34 \text { to } 0.02)\end{array}$ & NA & $\begin{array}{l}-0.57 \\
(-0.81 \text { to }-0.33)\end{array}$ & $\begin{array}{l}-0.55 \\
(-0.77 \text { to }-0.33)\end{array}$ & $\begin{array}{l}-0.05 \\
(-0.25 \text { to } 0.15)\end{array}$ & $\mathrm{NA}$ & $\begin{array}{l}-0.40 \\
(-0.68 \text { to }-0.12)\end{array}$ & $\begin{array}{l}-0.36 \\
(-0.6 \text { to }-0.12)\end{array}$ \\
\hline $\begin{array}{l}\text { Total nicotine } \\
\text { (nicotine/cigarette } \\
(\mathrm{mg}))\end{array}$ & NA & $\begin{array}{l}0.07 \\
(-0.09 \text { to } 0.23)\end{array}$ & $\begin{array}{l}0.48 \\
(0.26 \text { to } 0.70)\end{array}$ & $\begin{array}{l}0.50 \\
(0.30 \text { to } 0.70)\end{array}$ & NA & $\begin{array}{l}0.13 \\
(-0.05 \text { to } 0.31)\end{array}$ & $\begin{array}{l}0.41 \\
(0.15 \text { to } 0.67)\end{array}$ & $\begin{array}{l}0.45 \\
(0.23 \text { to } 0.67)\end{array}$ \\
\hline $\begin{array}{l}\text { Filter weight } \\
\quad(\mathrm{mg})\end{array}$ & NA & NA & NA & $\begin{array}{l}-0.16 \\
(-0.30 \text { to }-0.02)\end{array}$ & NA & NA & NA & $\begin{array}{l}-0.26 \\
(-0.42 \text { to }-0.10)\end{array}$ \\
\hline Intercept & 16.9 & 11.18 & 16.9 & 20.5 & 1.18 & 0.86 & 1.18 & 1.64 \\
\hline RMS error & 1.79 & 1.85 & 1.44 & 1.36 & 0.15 & 0.15 & 0.14 & 0.12 \\
\hline$R^{2}($ all $\mathrm{p}<0.0001)$ & 0.76 & 0.74 & 0.85 & 0.87 & 0.73 & 0.74 & 0.79 & 0.85 \\
\hline
\end{tabular}

NA = not applicable

Table 8 Beta weights (95\% confidence intervals) for multiple regression equations predicting standard tar and nicotine yields of Canadian cigarettes from percent ventilation, percent nicotine in tobacco, nicotine content per cigarette, and filter weight. Each column describes a different regression equation.

\begin{tabular}{|c|c|c|c|c|c|c|c|c|}
\hline \multirow[b]{2}{*}{ Percent ventilation } & \multicolumn{4}{|l|}{ Predicting tar yields } & \multicolumn{4}{|c|}{ Predicting nicotine yields } \\
\hline & $\begin{array}{l}-0.90 \\
(-1.11 \text { to }-0.69)\end{array}$ & $\begin{array}{l}-0.90 \\
(-1.11 \text { to }-0.69)\end{array}$ & $\begin{array}{l}-0.71 \\
(-0.94 \text { to }-0.48)\end{array}$ & $\begin{array}{l}-0.67 \\
(-0.86 \text { to }-0.48)\end{array}$ & $\begin{array}{l}-0.86 \\
(-1.13 \text { to }-0.59)\end{array}$ & $\begin{array}{l}-0.85 \\
(-1.10 \text { to }-0.60)\end{array}$ & $\begin{array}{l}-0.66 \\
(-0.95 \text { to }-0.37)\end{array}$ & $\begin{array}{l}-0.60 \\
(-0.87 \text { to }-0.33)\end{array}$ \\
\hline $\begin{array}{l}\text { Percent nicotine } \\
\text { (mg nicotine/mg } \\
\text { tobacco) }\end{array}$ & $\begin{array}{l}-0.01 \\
(-0.18 \text { to } 0.16)\end{array}$ & NA & $\begin{array}{l}-0.84 \\
(-1.48 \text { to }-0.20)\end{array}$ & $\begin{array}{l}-0.72 \\
(-1.26 \text { to }-0.18)\end{array}$ & $\begin{array}{l}0.07 \\
(-0.20 \text { to } 0.34)\end{array}$ & NA & $\begin{array}{l}-0.81 \\
(-1.63 \text { to } 0.01)\end{array}$ & $\begin{array}{l}-0.67 \\
(-1.39 \text { to } 0.05)\end{array}$ \\
\hline $\begin{array}{l}\text { Total nicotine } \\
\quad \text { (nicotine/ } \\
\text { cigarette) }(\mathrm{mg}))\end{array}$ & $\mathrm{NA}$ & $\begin{array}{l}0.05 \\
(-0.14 \text { to } 0.24)\end{array}$ & $\begin{array}{l}0.83 \\
(0.19 \text { to } 1.47)\end{array}$ & $\begin{array}{l}0.84 \\
(0.32 \text { to } 1.36)\end{array}$ & NA & $\begin{array}{l}0.13 \\
(-0.12 \text { to } 0.38)\end{array}$ & $\begin{array}{l}0.88 \\
(0.09 \text { to } 1.67)\end{array}$ & $\begin{array}{l}0.90 \\
(0.22 \text { to } 1.58)\end{array}$ \\
\hline Filter weight (mg) & NA & NA & NA & $\begin{array}{l}-0.27 \\
(-0.44 \text { to }-0.10)\end{array}$ & NA & NA & NA & $\begin{array}{l}-0.34 \\
(-0.57 \text { to }-0.11)\end{array}$ \\
\hline Intercept & 17.1 & 15.5 & 18.7 & 39.7 & 1.37 & 1.24 & 1.51 & 3.8 \\
\hline RMS error & 1.98 & 1.96 & 1.72 & 1.39 & 0.22 & 0.21 & 0.20 & 0.17 \\
\hline$R^{2}($ all $\mathrm{p}<0.0001)$ & 0.81 & 0.82 & 0.87 & 0.92 & 0.71 & 0.72 & 0.77 & 0.84 \\
\hline
\end{tabular}

CORRELATIONS

Tables 4 and 5 show the correlation matrices for the United States, Canadian, British and all brands combined. Machine-smoked yields of tar, nicotine, and $\mathrm{CO}$ were all very highly intercorrelated. Looking at associations with the yield measures, the most consistent finding across the three samples is the high correlation between filter ventilation and machine-smoked yields of tar, nicotine, and CO. Next, filter weight was reliably associated with machinesmoked yields, especially in the American sample. American cigarettes with higher machinesmoked yields had a somewhat higher total nicotine content, but no higher nicotine concentration in the tobacco. British cigarettes with lower machine-smoked yields were likely to have a higher percentage of nicotine in the tobacco, but not likely to have higher total nicotine content.

MULTIPLE REGRESSION ANALYSES

Tables 6, 7, and 8 show the results of the regression analyses predicting machinesmoked yields of tar and nicotine. (Very similar patterns of effects were found in regression equations predicting machine-smoked yields of CO; they are not reported to save space.) Total nicotine and percent nicotine give evidence of acting as "suppressor variables." A suppressor variable is a predictor variable that is highly intercorrelated with another predictor variable, and this intercorrelation "suppresses" variance that is not related to the prediction of the dependent variable. Because of this possible suppressor effect, it would be wrong to conclude that when both variables are in the model that their significant standardised coefficients reflect the direct contributions of these variables as predictors. ${ }^{14}$ Notwithstand- ing, when percent nicotine and total nicotine are both included in models, there is an indication that as machine-smoked yields of tar and nicotine decrease, percent nicotine increases and total nicotine decreases. Across all models and countries, ventilation was an important predictor variable (see tables 4-8) with simple correlations between ventilation and machinesmoked yields ranging from about 0.80 to 0.90 and with multivariate analyses showing standardised regression coefficients from about 0.60 to 0.90 for ventilation predicting machine-smoked yields.

\section{Discussion}

Ventilation appears to be the major method of manipulating machine-smoked yields of tar, nicotine, and $\mathrm{CO}$ in three countries. On no pack was the presence of vents indicated. On none of the filters were the location of vents marked with a special marking such as a coloured band, though coloured bands and logos were found on most cigarettes, indicating the feasibility of marking vents. Filter vents can be invisible to the naked eye; magnifiers, bright lights, and dissection of the filter were often necessary to locate them. Smokers are more likely to be ignorant of the presence of invisible vents than visible vents. ${ }^{10}$ Filter ventilation is particularly important because smokers can defeat filter ventilation. Unless the smoker removes the filter entirely, the filtration effects related to filter weight should be relatively unchanged by behaviour.

Recently released industry documents are consistent with the view that invisible vents may have been part of a research and development strategy for some manufacturers. In a BAT document ( $R$ \& $D$ views on potential marketing opportunities), point 1 entitled: "Elastic/ 
compensatible products," in a section labelled "high priority," notes: "Irrespective of the ethics involved, we should develop alternative designs (that do not invite obvious criticism) which allow the smoker to obtain significant enhanced deliveries should he so wish". ${ }^{15}$ A Brown \& Williamson document notes that in designing a product for compensation (giving more to the smoker than the standard tests indicate) "designing to the subconscious is preferred to requiring the smoker to commit a conscious act". ${ }^{16}$ In another BAT document, the answer is given to the question, "Which product/design properties influence elasticity? 1. Tip ventilation: bigger effects at higher degree of ventilation. . . 2. Delivery of the blend". ${ }^{17}$

We generally support Benowitz's conclusions that low standard tar and nicotine yields are found in cigarettes that have within their tobacco column ample doses of nicotine. ${ }^{2}$ Cigarettes with low machine-smoked nicotine yields are not, therefore, low nicotine content cigarettes.

Many smokers who use brands with ventilated filters will block some of those vents while smoking. ${ }^{3}$ Lip blocking would more likely occur with the brands with shorter vent distances, ${ }^{10}$ and finger blocking with those with longer distances. One study has estimated that $58 \%$ of persons who smoke cigarettes with machine-smoked yields of less than $6 \mathrm{mg}$ tar are blocking some of the filter vents. ${ }^{4}$ Inadvertently blocking even a portion of the filter vents can markedly increase a smoker's exposure to the harmful components of cigarette smoke. In tests conducted on smoking machines, blocking half of the ventilation holes on a cigarette that had machine-smoked yields of $4 \mathrm{mg}$ tar, $0.5 \mathrm{mg}$ nicotine, and $5 \mathrm{mg} \mathrm{CO}$ increased tar yields by $60 \%$, nicotine by $62 \%$, and CO by $73 \%$. $^{5}$ One study from the tobacco industry ${ }^{3}$ estimated that $45 \%$ of smokers, when smoking an Ultra-light cigarette $(2.2 \mathrm{mg}$ tar), blocked vents to some degree with lips: $21 \%$ of smokers (or nearly half of those who block) increased tar yields to at least $3.3 \mathrm{mg} \operatorname{tar}$ (that is, by $50 \%$ or more); overall one in 10 smokers (about $25 \%$ of those who block) were estimated to at least double their tar yields from lip blocking alone.

Take a closer look at one Canadian brand family: Players. Players had a 28\% market share in 1995 in Canada. They were indeed best-sellers. Inspection of table 2 shows that Players Extra Light cigarettes in Canada have quite a high filter ventilation level $(42 \%)$ for the $1.2 \mathrm{mg}$ nicotine yield brand, but this is coupled with a very high total nicotine content (18.3 mg per cigarette), whereas the Players Full Flavor were unventilated at $1.4 \mathrm{mg}$ nicotine yield and had a nicotine content of only $8.0 \mathrm{mg}$ per cigarette. Imperial Tobacco, the makers of Players, has conducted large-scale studies of vent-blocking with lips. For example, a 1997 study by Imperial involved the collection of 2232 cigarette butts and the measurement of mouth-insertion depth; if the vents were positioned at $12 \mathrm{~mm}$ on the filter (as for Players Extra Light), the table 3 in their published study shows that about one in four smokers (28\%) would block at least some of the vent holes with their lipsthree in 100 smokers were shown by the manufacturer's data, to perform "complete hole coverage" with their lips given this vent positioning.

\section{LIMITATIONS}

The American data on machine-smoked yields may be outdated. The most recently released FTC scores are from cigarettes purchased in 1995. Sampling of cigarettes was not random or sales weighted. Standard temperature and humidity conditioning of cigarettes before testing for ventilation or nicotine content might have reduced variation because of temperature/ humidity conditions prevailing at the site of purchase of the cigarettes. Testing freshly opened packs provides a simulation of what a consumer might encounter. Though the temperature/humidity conditions of cigarette storage before nicotine testing were not controlled, they were common for all the brands tested. Since temperature and humidity will affect the weight of tobacco, our testing and storage conditions might reduce the exact comparability of our absolute nicotine values with results from other laboratories-but the relative values for nicotine content of tobacco should be highly consistent. Other extraction procedures and different analytical procedures, such as gas-liquid chromatography, might produce somewhat different results.

Notwithstanding, there is evidence from other datasets that our filter ventilation measures were excellent and our nicotine measures were good. Data submitted to the Massachusetts Department of Health by American cigarette manufacturers could be matched for 10 brand formulations that we report on here. Two additional matches could be made by using data not reported here that had been collected by us on the new Winston brands. For these 12 brands, the $r^{2}$ for the association of our scores with industry scores for percent filter ventilation was $0.99(p<0.0001)$ and for total nicotine was $0.67(\mathrm{p}=0.001)$. Three of the brands were tested using different analytical procedures than those specified by the Massachusetts Department of Public Health by Philip Morris. Limiting the analyses to those nine brands employing the same procedures, the $r^{2}$ for total nicotine was 0.81 ( $\mathrm{p}=$ 0.001 ). RJ Reynolds contracted with Labstat to test their 33 brands using the same protocols and techniques: the $r^{2}$ for these analyses was 0.85 ( $p<0.0001)$. The RJ Reynolds values were $1.12 \mathrm{mg}$ lower than the Labstat measures $(\mathrm{df}=$ $32, t=12.11, \mathrm{p}<0.0001)$; our values were $0.96 \mathrm{mg}$ lower than the Massachusetts scores ( $\mathrm{df}=8, t=3.02, \mathrm{p}=0.02)$ These analyses provide evidence that our nicotine tests provide useful measures that are about as good as can be found with two practiced tobacco laboratories using standard temperature and humidity conditions and the same protocols. 
STANDARD TESTS

Standard cigarette testing needs to include nicotine content (as in the new Massachusetts tests), percent nicotine (as is missing from the Massachusetts test), as well as the number of puffs taken in any smoking machine procedure. ${ }^{18}{ }^{19}$ The level of precision and accuracy needed in cigarette testing needs to be judged in relation to the variation in human smoking behaviour. For example, there is little reason to believe that a mid-ranking difference of $2 \mathrm{mg}$ tar, $0.2 \mathrm{mg}$ nicotine, and $2 \mathrm{mg} \mathrm{CO}$ will be reflected in any measurable differences in exposures to human smokers.

In the United States a voluntary agreement between the Federal Trade Commission and the tobacco industry provides for machinesmoked cigarette testing and the reporting of these results in advertising. ${ }^{1}$ Research has shown, however, that few smokers even know to look in advertising for such information, and further "non-advertised" generic brands are generally not advertised at all. ${ }^{20}$ It is important to understand that, in the United States, machine-smoked tar and nicotine yields are not required to be placed on cigarette packs, whereas in Canada and the United Kingdom, machine-smoked yields are included on cigarette packs.

We did not measure all variables that may influence delivery of smoke toxins. For example, paper porosity can also alter air dilution of smoke, and chemicals can also alter the burn rate of a tobacco column, influencing the number of puffs taken in a standard assay. Filter efficiency (based on materials and length) and length of filter overwrap could also influence machine-smoked yields. ${ }^{21}$ Smoke $\mathrm{pH}$ could also alter the effects of machine-smoked nicotine yields. ${ }^{22}$

Machine-smoked yields of tar and nicotine have little relevance for smokers' actual intakes, and through brand identifiers, such as "Light" and "Ultralight" may be actively misleading. ${ }^{20}{ }^{23}$ Our data show that the combination of ample nicotine content with filter vents that are easily, and often unconsciously defeated by smokers provides a readily available means for smokers to satisfy their addiction to nicotine from even low-yield brands. Numerous studies indicate extensive nicotine compensation in smokers of low nicotine cigarettes. ${ }^{2425}$

Filter ventilation appears to be the predominant method for reducing machine-smoked yields of tar, nicotine, and $\mathrm{CO}$ in three countries. Some cigarettes contain about twice as much nicotine (total content or percent nicotine) as do other brands, indicating that tobacco types or blends and tobacco casings can substantially manipulate nicotine content of cigarettes. It is lamentable that consumers or scientists routinely know so little about so common a consumer "good" that causes so much death and disability. The more the cigarette is allowed to be a "black box"-even to government chemists-the easier it would be to fool most of the people most of the time, if an unscrupulous manufacturer chose to do so.

This study was supported by subcontract number TS 257-13/13 under the cooperative agreement between the Association of Teachers of Preventive Medicine and the Centers for Disease Control and Prevention. Thanks for helpful comments on the manuscript go to Gary A Giovino.

1 Rodgman A. FTC "tar" and nicotine in mainstream smoke: a retrospective. Recent Adv Tobacco Sci 1997;23:5-74

2 Benowitz NL, Hall SM, Herning RI, et al. Smokers of lowyield cigarettes do not consume less nicotine. $N$ Engl f Med 1983;309:139-42

3 Baker RR, Lewis LS. Filter ventilation-has there been a "cover up"? Recent Adv Tobacco Sci 1997;23:152-96.

4 Kozlowski LT, Pope MA, Lux JE. Prevalence of the misuse of ultra-low-tar cigarettes by blocking filter vents. Am $\mathcal{F}$ Public Health 1988;78:694-5.

5 Kozlowski LT, Frecker RC, Khouw V, et al. The misuse of 'less-hazardous' cigarettes and its detection: hole-blocking of ventilated filters. Am f Public Health 1980;70:1202-3.

6 Kozlowski LT, Pillitteri JL, Sweeney CT. Misuse of "Light" cigarettes by means of vent blocking. F Substance Abuse 1994;6:333-6.

7 Kozlowski LT, Heatherton TF, Frecker RC, et al. Selfselected blocking of vents on low-yield cigarettes. Pharmacol Biochem Behav 1989;33:815-19.

8 Kozlowski LT, Sweeney, CT, Pillitteri JL. Blocking cigarette filter vents with lips more than doubles carbon monoxide intake from ultra-low tar cigarettes. Exp Clin Psychopharmacol 1996;4:1-5

9 Kozlowski LT, Goldberg ME, Yost BA, et al. Smokers are unaware of the filter vents now on most cigarettes: results of a national survey. Tobacco Control 1996;5:265-70.

10 Kozlowski LT, White EL, Sweeney CT, et al. Few smokers know their own cigarettes have filter vents. $A m \mathcal{F} P u b$ Health 1998;88:681-2

11 Rickert WS, Robinson JC, Young JC, et al. A comparison of the yields of tar, nicotine, and carbon monoxide of 36 brands of Canadian cigarettes tested under three condibrands of Canadian cigarettes teste
tions. Prev Med 1983;12:682-94.

12 US Centers for Disease Control and Prevention. Filter ventilation levels in selected US cigarettes, 1997. MMWR tilation levels in

13 Mendenhall W, Sincich T. A second course in statistics: regression analysis. Upper Saddle River, New Jersey: PrenticeHall, 1996

14 Smith RL, Ager JW, Williams DL. Suppressor variables in multiple regression/correlation. Educ Psychol Measurement 1992;52:17-29

15 BAT Co Ltd. R\&D views on potential marketing opportunities. (Labelled "not for circulation", 12 September 1984.$)$ Minnesota Trial Exhibit 11275. <www.mnbluecrosstobacco.com>.

16 Sanford RA. Research program, future products. (Internal correspondence to Kohnhorst EE, Brown \& Williamson Tobacco Corporation, June 28 1985.) Minnesota Trial Exhibit 13250 . <www.mnbluecrosstobacco.com>.

17 Pangritz O, Smoke elasticity. Session III, Proceedings of the Smoking Behaviour-Marketing Conference, BAT Co Ltd., Montreal, Quebec, 9-12 July 1984:58. Minnesota Trial Montreal, Quebec, 9-12 July 1984:58. Minneso

18 Kozlowski LT. Tar and nicotine delivery of cigarettes: what a difference a puff makes. $7 A M A$ 1981;245:158-9.

19 Kozlowski LT, Rickert W, Robinson J, et al. Have tar and nicotine yields of cigarettes changed? Science 1980; 209:1550-1.

20 Kozlowski LT, Pillitteri JL, Yost BA, et al. Advertising fails to inform smokers of official tar yields of cigarettes. $\mathcal{F} A p p l$ Biobehav Res 1998;3:55-64.

21 Grunberg NE, Morse DE, Maycock VA, et al. Changes in overwrap and butt length of American cigarettes. An influence on reported tar yields. New York State $\mathcal{f}$ Med 1985;85:310-12.

22 Kessler DA, Witt AM, Barnett PS, et al. The Food and Drug Administration's regulation of tobacco products. $N$ Engl $\mathcal{F}$ Med 1996:335:988-94.

23 Kozlowski LT, Goldberg ME, Yost BA, et al. Smoker misperception of light and ultra-light cigarettes may keep them smoking. Am $\mathcal{F}$ Prev Med 1998;15:9-16.

24 Russell MAH, Jarvis MJ, Feyerabend C, et al. Reduction of tar, nicotine and carbon monoxide intake in low tar smokers. F Epidemiol Commun Health 1986;40:80-5.

25 Withey CH, Papacosta AO, Swan AV, et al. Respiratory effects of lowering tar and nicotine levels of cigarettes smoked by young male middle tar smokers. II: Results of a randomized controlled trial. $\mathcal{F}$ Epidemiol Commun Health 1992;46:281-5. 